\section{OP05 EVALUATING SOCIAL AND SPATIAL INEQUALITIES OF LARGE SCALE RAPID LATERAL FLOW SARS-COV-2 ANTIGEN TESTING IN COVID-19 MANAGEMENT: AN OBSERVATIONAL STUDY OF LIVERPOOL, UK (NOVEMBER 2020 TO JANUARY 2021)}

${ }^{1}$ Mark Green*, ${ }^{2}$ Marta Garcia-Finana, ${ }^{3}$ Ben Barr, ${ }^{2}$ Girvan Burnside, ${ }^{2}$ Chris Cheyne, ${ }^{2}$ David Hughes, ${ }^{4}$ Matthew Ashton, ${ }^{3}$ Sally Sheard, ${ }^{3}$ Iain Buchan. ' Geography and Planning, University of Liverpool, Liverpool, UK; ${ }^{2}$ Health Data Science, University of Liverpool, Liverpool, UK; ${ }^{3}$ Public Health and Policy, University of Liverpool, Liverpool, UK; ${ }^{4}$ Public Health, Liverpool City Council, Liverpool, UK

\subsection{6/jech-2021-SSMabstracts.5}

Background Asymptomatic transmission of SARS-CoV-2 poses a significant burden on managing the spread of COVID-19. Few studies have evaluated the impact of testing for asymptomatic COVID-19 among large populations or whole cities using empirical data. No study to our knowledge has considered if such interventions result in or exacerbate existing socioeconomic inequalities. The aim of our study is to explore social and spatial inequalities in uptake and case-detection of rapid lateral flow SARS-CoV-2 antigen tests (LFTs) offered to people without symptoms of COVID-19 in Liverpool between 6th November 2020 and 31st January 2021.

Methods Linked pseudonymised records for asymptomatic residents in Liverpool (UK) who received a LFT for COVID-19 between 6th November 2020 to 31st January 2021 were accessed using the Combined Intelligence for Population Health Action (CIPHA) data resource. Bayesian Hierarchical Poisson Besag, York, and Mollié models were used to estimate ecological associations for uptake and positivity of testing.

Results 214525 residents (43\%) received a LFT identifying 5557 individuals as positive cases of COVID-19 (1.3\%). Uptake was highest in November when there was military assistance. High uptake was observed again in the week preceding Christmas and was sustained into a national lockdown. Overall uptake and repeat testing were lower among males (e.g. $40 \%$ uptake over the whole period), Black Asian and other Minority Ethnic groups (e.g. 27\% uptake for 'Mixed' ethnicity) and in the most deprived areas (e.g. 32\% uptake in most deprived areas). These population groups were also more likely to have received positive tests for COVID-19. Models demonstrated that uptake and repeat testing were lower in areas of higher deprivation, areas located further from test sites and areas containing populations less confident in the using Internet technologies. Positive tests were spatially clustered in deprived areas.

Conclusion Our study provides the first substantial evidence on inequalities involved in large-scale asymptomatic rapid testing of populations for SARS-CoV-2. Large-scale voluntary asymptomatic community testing saw social, ethnic, and spatial inequalities in an 'inverse care' pattern, but with an added digital exclusion factor. While test uptake was popular, there was a disconnect between the populations accessing testing and those experiencing harms relating to COVID-19. COVID19 testing and support to isolate need to be more accessible to the vulnerable communities most impacted by the pandemic, including non-digital means of access.

\section{OP06 DIFFERENCES IN THE RATES OF NEW DIAGNOSES REPORTED PRE AND DURING THE COVID-19 PANDEMIC}

Jennifer Cole*, Shivan Thakrar, Helen M Parretti, Nick Steel. Department of Medicine, University of East Anglia, Norwich, UK

10.1136/jech-2021-SSMabstracts. 6
Background Excess deaths from conditions other than COVID-19 have been reported during the pandemic. Difficulty accessing healthcare is a possible explanation. We aimed to assess changes in rates of new diagnoses for common conditions before and during the pandemic.

Methods Participants aged 50 years and older in three waves of the English Longitudinal Study for Ageing were asked whether a doctor had told them that they had newly developed any of 11 medical conditions: hypertension, angina or heart attack, heart failure, diabetes, stroke, chronic lung disease, asthma, arthritis, cancer, dementia, or blood disorder. Responses were compared between pre-COVID Wave 9 (W9) in 2018 to 2019, COVID Wave 1 (CW1) in June and July 2020, and COVID Wave 2 (CW2) in November and December 2020. Multivariate logistic regression on weighted data was performed to assess variation by sex, age group (50 to state pension age (SPA), SPA to 74,75 and over), and wealth quintile.

Results The overall rate of new diagnoses reported per 1,000 participants per year was 116.76 in W9 $(n=7,289), 110.10$ in CW1 $(\mathrm{n}=5,825)$, and 138.23 in CW2 $(\mathrm{n}=5,339)$. Diagnosis rates dropped from W9 to CW1 to CW2 for dementia (9.09 to 4.01 to 2.25 respectively), angina and heart attack (13.50 to 11.67 to 10.86 ), and stroke (7.05 to 6.18 to 4.12 ). In contrast, the rate increased for arthritis (29.11 to 37.42 to 44.58 ) and diabetes (18.83 to 21.06 to 23.60 ). Multivariate analysis found that increasing age was a predictor for dementia diagnosis in cw2 (odds ratio 2.20; 95\% confidence interval 1.12 to 4.30). No other regression analyses were statistically significant.

Conclusion Drops in diagnosis rates for dementia and cardiovascular disease during the pandemic could be contributing to increased deaths from conditions other than COVID-19. Policy initiatives are underway to encourage people to seek healthcare as normal, and it will be important to maintain adequate access to healthcare for major conditions in any future lockdown. The reasons for the reported substantial rise in arthritis and smaller rise in diabetes diagnosis rates are not clear and require further investigation.

Wednesday 15 September

CVD \& CVD Modelling, $13.00-15.25$

\section{OP07 ASSOCIATION BETWEEN CARDIOVASCULAR HEALTH AND HEART FAILURE IN OLDER BRITISH MEN; FINDINGS FROM THE BRITISH REGIONAL HEART STUDY}

${ }^{1}$ Mehar Chawla*, 'Olia Papacosta, 'Lucy Lennon, ${ }^{2}$ Peter Whincup, 'S Goya Wannamethee. ${ }^{1}$ Department of Primary Care and Population Health, University College London, London, UK; ${ }^{2}$ Population Health Research Institute, St George's University of London, London, UK

\subsection{6/jech-2021-SSMabstracts.7}

Background With an ageing population heart failure (HF) is emerging as a common cardiovascular condition and is one of the leading causes of mortality and morbidity. The American Heart Association promotes Life simple's 7 (LS7) to improve cardiovascular health (CVH). LS7 consists of seven modifiable health factors: body mass index, blood pressure, glucose, cholesterol, physical activity, smoking and diet. We sought to examine the impact of LS7 on risk of incident HF in older British men as well as the overall 